\title{
Is Becker dystrophinopathy a contraindication to heart transplant?: experience in a single institution
}

\author{
¿La distrofinopatía de Becker es una contraindicación para el trasplante \\ cardiaco?: experiencia de un centro
}

\author{
Marisol Guadalupe Ascencio-Lemus, ${ }^{a}$ Eduardo Barge-Caballero, ${ }^{\text {b,c }}$ María J. \\ Paniagua-Martín, ${ }^{\text {,cc }}$ Gonzalo Barge-Caballero, ${ }^{\text {bcc }}$ David Couto-Mallón, ${ }^{\text {bcc }}$ María G. \\ Crespo-Leiro ${ }^{\mathrm{b}, \mathrm{c}}$

\footnotetext{
${ }^{a}$ Servicio de Cardiología, Complejo Asistencial Universitario de León, León, Spain

${ }^{b}$ Unidad de Insuficiencia Cardiaca y Trasplante Cardiaco, Servicio de Cardiología, Complejo Hospitalario Universitario de A Coruña (CHUAC), Instituto de Investigación Biomédica de A Coruña (INIBIC), A Coruña, Spain
} \\ ${ }^{c}$ Centro de Investigación Biomédica en Red de Enfermedades Cardiovasculares (CIBERCV), Madrid, Spain
}

To the Editor,

Becker dystrophinopathy is an X-linked inherited disease caused by a mutation in the Xp21 gene, which encodes dystrophin. ${ }^{1}$ Dystrophin is expressed in both skeletal and cardiac muscle, and the disease can thus feature myopathic and cardiovascular symptoms.

Clinical manifestations generally appear in the second decade of life and usually begin with muscle weakness in the pelvic girdle. ${ }^{2}$ Cardiac involvement is an infrequent manifestation, but affects some patients. 3,4 The most frequent cardiac manifestation in these patients is dilated cardiomyopathy. $^{2}$

Becker dystrophinopathy patients with refractory heart failure are candidates for heart transplant (HTx), but there is some debate about the suitability of this procedure. ${ }^{1,3,4}$ The main concerns about HTx for Becker dystrophinopathy patients are the potential perioperative risk, the risk that HTx might exclude patients from subsequent respiratory physiotherapy and motor rehabilitation, and the risk that post-HTx corticosteroid therapy will trigger myopathy progression. This article presents a single-center experience with a small series of Becker dystrophinopathy patients with dilated cardiomyopathy who underwent HTx. 
From 1991 to 2017,781 patients received HTx at the study center. Of these patients, $6(0.8 \%)$ had a diagnosis of dilated cardiomyopathy associated with Becker muscular dystrophy. The baseline clinical characteristics of these patients are summarized in Table 1.

Table 1. Baseline Characteristics

\begin{tabular}{|c|c|c|c|c|c|c|}
\hline Patient & Sex & Age at NMD onset, $y$ & Age at HF onset, $y$ & Age at HTx, y & Pre-HTx CK, IU/L & Follow-up, mo \\
\hline 1 & Male & 9 & 41 & 44 & 37 & 230 \\
\hline 2 & Male & 4 & 20 & 24 & 775 & 159 \\
\hline 3 & Male & 12 & 35 & 43 & 416 & 91 \\
\hline 4 & Male & 15 & 30 & 36 & 257 & 77 \\
\hline 5 & Male & 28 & 35 & 44 & 445 & 12 \\
\hline 6 & Male & 46 & 30 & 48 & 342 & 10 \\
\hline
\end{tabular}

CK, creatine kinase; HF, heart failure; HTx, heart transplant; NMD, neuromuscular disease.

The median age of patients at the time of dystrophinopathy diagnosis was 13.5 years. Clinical manifestation of heart failure occurred at a median age of 32.5 years, and patients underwent HTx at a median age of 43.5 years. HTx was an emergency procedure in 4 patients $(67 \%)$. At the time of inclusion on the waiting list, all patients had mild muscle damage and complete functional autonomy, with no significant respiratory involvement.

All the patients received immunosuppressive therapy with calcineurin inhibitors (cyclosporine or tacrolimus) and mycophenolate mofetil, except for 1 patient who received azathioprine. All patients were placed on indefinite corticosteroid therapy, with the dose reduced as much as possible from the sixth month post-HTx (target prednisone maintenance dose $\leq 5 \mathrm{mg} / \mathrm{d}$ ). Statin therapy was prescribed in all patients but was withdrawn in 2 patients $(33.3 \%)$ due to muscle toxicity featuring marked elevation of serum creatine kinase.

In the immediate postoperative period, 3 patients (50\%) developed acute renal failure requiring dialysis. In the same period, 2 patients $(33 \%)$ developed posterior reversible encephalopathy syndrome linked to calcineurin inhibitors, and 1 patient $(17 \%)$ developed severe resistant hypertension. Extubation was performed within 24 hours of surgery in 2 of the 6 patients (33\%) and was maintained for between 5 and 13 days after transplant surgery in the remaining 4 patients. There were no instances of immediate postoperative death or episodes of early transplant dysfunction requiring mechanical circulation support.

All patients had long-term survival after HTx over a mean 96-month follow-up (range, 10-230 months). At the time of writing, there has been only 1 death, due to lung cancer and occurring 19 years after the transplant surgery. Transplanted organ function is conserved in the 5 surviving patients, and to date there have been no episodes of transplant rejection with hemodynamic deterioration. Slow post-HTx progression of muscle damage has resulted in 3 patients needing a wheelchair for mobility.

The medical literature contains few reports of Becker muscular dystrophy patients undergoing HTx; however, the little available evidence indicates that these patients have similar long-term outcomes to the general population of heart transplant recipients with other heart conditions. ${ }^{4}$ 
The results observed in this cohort of heart transplant recipients with Becker dystrophinopathy are satisfactory, with long-term survival and good functional prognosis indicating well-tolerated immunosuppressive therapy. HTx thus appears to be a valid option for Becker dystrophinopathy patients with refractory heart failure who show relatively preserved extracardiac muscle function before the intervention.

\section{REFERENCES}

1. Papa AA, Ambrosio PD, Petillo R, Palladino A, Politano L. Heart transplantation in patients with dystrophinopathic cardiomyopathy: Review of the literature and personal series. Intractable Rare Dis Res. 2017;6:95-101.

2. Finsterer J, Sto “ llberger C. Cardiac involvement in Becker muscular dystrophy. Can J Cardiol. 2008;24:786-792.

3. Ruiz-Cano MJ, Delgado JF, Jimenez C, et al. Successful heart transplantation in patients with inherited myopathies associated with end-stage cardiomyophaty. Transplant Proc. 2003;35:1513-1515.

4. Wu RS, Gupta S, Brown RN, et al. Clinical outcomes after cardiac transplantation in muscular dystrophy patients. J Heart Lung Transplant. 2010;29:432-438. 